\title{
Recent advances on machine learning and Cybernetics
}

\author{
Witold Pedrycz $\cdot$ Daniel Yeung $\cdot$ Xizhao Wang
}

Published online: 6 March 2010

(C) Springer-Verlag 2010

During the past decades, the developments of machine learning and Cybernetics realized in the framework of soft computing techniques such as rough sets, neural networks, evolutionary algorithms, fuzzy logic, support vector machines and Granular Computing have enjoyed a considerable visibility. We have been witnessing a wealth of interesting pursuits when it comes to the fundamentals of machine learning and Cybernetics as well as their numerous applications.

This special issue of the Soft Computing Journal reports on some recent advances in the theory and applications of soft-computing-based machine learning and Cybernetics as well as their synergistic undertakings.

The papers included in this special issue have been carefully selected from the submissions to the 7 th International Conference on Machine Learning and Cybernetics (ICMLC2008) held in Kunming, China, on July 2008. In total, the ICMLC2008 received 1,542 submissions and after a thorough review 750 papers were accepted for presentation. A number of best papers on soft-computingbased machine learning and cybernetics were afterwards

W. Pedrycz $(\bowtie)$

University of Alberta, Edmonton, Canada

e-mail: pedrycz@ee.ualberta.ca

W. Pedrycz

Systems Research Institute, Polish Academy of Sciences,

Warsaw, Poland

D. Yeung

Machine Learning Institute, Kowloon, Hong Kong

e-mail: csdaniel@inet.polyu.edu.hk

X. Wang

Hebei University, Hebei, China

e-mail: xizhaowang@ieee.org selected and the authors were advised to submit to this special issue. All the papers have been thoroughly refereed by experts who evaluated them based on the criteria of originality, significance, quality, and clarity. As a result, there are 16 papers accepted for publication in this special issue.

As stressed by the title of this special issue, the original papers are focused on the research issues of the fusion of machine learning and cybernetics being cast in the framework of soft computing. Several contributions elaborate on new machine learning theories, technologies, and algorithms including those coming from supervised learning, unsupervised learning, semi-supervised learning, reinforcement learning, and kernel-based learning. With reference to soft computing, the papers concentrate on exploring the theoretical foundations and applied approaches to uncertainty processing pertaining to learning and data mining. These studies may help establish important linkages between the field of machine learning and other research domains. Several contributions elaborate on realworld applications of machine learning and cybernetics to medical image processing and business intelligence.

The Guest Editors issue would like to take this opportunity and express their sincere thanks to the reviewers for their detailed comments as well as constructive suggestions on how to improve the quality of the manuscripts. We would like to thank the Editor-in-Chief of SOFT COMPUTING for the encouragement, support and guidance during the realization of this special issue.

\section{Guest Editors}

Witold Pedrycz

Daniel Yeung

Xizhao Wang 